\title{
Molecular epidemiology of Sporothrix schenkii isolates in Malaysia
}

\begin{abstract}
Sporothrix schenkii is a dimorphic fungus that causes infections in both humans and animals. We report on $25 \mathrm{~S}$. schenkii isolates collected in 2017 from humans and cats clinically diagnosed with sporotrichosis, in Malaysia. These isolates were phenotypically identified as $\mathrm{S}$. schenkii sensu lato and further defined as S. schenckii sensu stricto based on partial calmodulin gene sequence. Isolates from both humans and cats were genotypically identical but displayed phenotypic variation. Phylogenetic analyses based on partial calmodulin sequence showed that the Malaysian isolates clustered with global S. schenkii sensu stricto strains, in particular, of the AFLP type E. This analysis also revealed that partial calmodulin sequence alone was sufficient for classifying global S. schenckii sensu stricto strains into their respective AFLP types, from A to E. The genetically conserved S. schenkii sensu stricto species isolated from humans and cats is suggestive of a clonal strain present in Malaysia. To the best of our knowledge, this is the first report on molecular identification of Sporothrix schenkii strains from human infections in Malaysia. Further studies are required in order to elucidate the clonal nature of Malaysian S. schenkii isolates. Our findings indicate the presence of a predominant S. schenkii genotype in the environment, causing infections in both cats and humans in Malaysia.
\end{abstract}

Keyword: Sporothrix schenkii; Malaysia; Molecular identification; Calmodulin gene; Phylogenetic analysis 